\title{
RESEARCH
}

Open Access

\section{Mesenchymal stromal cells modulate the molecular pattern of healing process in tissue-engineered urinary bladder: the microarray data}

Marta Pokrywczynska* (D), Marta Rasmus, Arkadiusz Jundzill, Daria Balcerczyk, Jan Adamowicz, Karolina Warda, Lukasz Buchholz and Tomasz Drewa

\begin{abstract}
Background: Molecular mechanisms underlying the regenerative process induced by stem cells in tissue-engineered urinary bladder are poorly explained. The study was performed to explore the pathways associated with regeneration process in the urinary bladder reconstructed with adipose tissue-derived mesenchymal stromal cells (ASCs).

Methods: Rat urinary bladders were reconstructed with bladder acellular matrix (BAM) $(n=52)$ or BAM seeded with adipose tissue-derived mesenchymal stromal cells (ASCs) $(n=52)$. The process of bladder healing was analyzed at 7, 30 , 90, and 180 days postoperatively using macroscopic histologic and molecular techniques. Gene expression was analyzed by microarrays and confirmed by real-time PCR.

Results: Numerous differentially expressed genes (DEGs) were identified between the bladders augmented with BAM seeded with ASCs or BAM only. Pathway analysis of DEGs allows to discover numerous pathways among them Hedgehog, TGF- $\beta$, Jak-STAT, PI3-Akt, and Hippo modulated by ASCs during the healing process of tissue-engineered urinary bladder. Real-time PCR analysis confirmed upregulation of genes involved in the Hedgehog signaling pathway including Shh, Gli1, Smo, Bmp2, Bmp4, Wnt2, Wnt2b, Wnt4, Wnt5a, and Wnt10 in urinary bladders reconstructed with ASC-seeded grafts.

Conclusion: The study provided the unequivocal evidence that ASCs change the molecular pattern of healing in tissue-engineered urinary bladder and indicated which signaling pathways triggered by ASCs can be associated with the regenerative process. These pathways can be used as targets in the future studies on induced urinary bladder regeneration. Of particular interest is the Hedgehog signaling pathway that has been upregulated by ASCs during healing of tissue-engineered urinary bladder.
\end{abstract}

Keywords: Urinary bladder, Healing, Regeneration, Regenerative pathways, Tissue engineering, Adipose tissue derived mesenchymal stromal cells, Microarrays, Hedgehog signaling pathway

\footnotetext{
* Correspondence: marta.pokrywczynska@interia.pl

Department of Regenerative Medicine, Cell and Tissue Bank, Chair of

Urology, Nicolaus Copernicus University in Torun, Ludwik Rydygier Medical

College in Bydgoszcz, 85-094, Marii Sklodowskiej Curie 9 Street, 85-094

Bydgoszcz, Poland
}

(c) The Author(s). 2019 Open Access This article is distributed under the terms of the Creative Commons Attribution 4.0 International License (http://creativecommons.org/licenses/by/4.0/), which permits unrestricted use, distribution, and reproduction in any medium, provided you give appropriate credit to the original author(s) and the source, provide a link to the Creative Commons license, and indicate if changes were made. The Creative Commons Public Domain Dedication waiver (http://creativecommons.org/publicdomain/zero/1.0/) applies to the data made available in this article, unless otherwise stated. 


\section{Background}

Tissue engineering of the urinary bladder is a fastdeveloping field of regenerative medicine [1]. Despite the lack of successful solutions being translated into clinical practice, tissue engineering of the urinary bladder is constantly making progress. New reconstructive therapies for urologists are reflected through the large amount of new research evaluating the latest stem cell and biomaterial science achievements for induced bladder regeneration. In reality reconstructive urology is still based on the approach established at the end of the nineteenth century, i.e., utilizing bowel wall as a replacement for urinary tracts. Therefore, known surgical techniques are reaching their limits in terms of a functional result that might be improved upon by gradual implementation of tissue engineering solutions [2]. In this context, tissue engineering seems to be a natural path for reconstructive urology development and might overcome the increasing stagnation in this field. Principles of bladder tissue engineering have been established a decade after the landmark research by Atala et al., which drew the urology community's attention on opportunities offered by regenerative medicine [3]. Stem cells, especially mesenchymal stem cells (MSCs), are the most commonly used cell population in tissue engineering, which can deliver paracrine factors that rearrange the local healing response $[4,5]$. Numerous studies indicated that stem cells promote regeneration of tissueengineered urinary bladder preventing fibrosis and scar formation [4-12]. Regeneration outcomes were found to be strictly dependent on the number of cells used for the bladder reconstruction [12]. Nevertheless, the exact mechanism by which stem cells trigger the underlying regenerative process is still poorly understood. Stem cell paracrine stimuli can promote healing by activation of regenerative pathways that have been silenced during ontogenesis. Regeneration is a complex process that is the result of actions from multiple signaling pathways that are linked together and activated in a synchronized manner. The Holy Grail of regenerative medicine is to define regenerative pathways and to develop effective methods of gene switching to direct tissue healing from repair to regeneration. Theoretically, postnatal tissue regeneration should recapitulate embryonic organogenesis; thus, the same cellular and molecular mechanisms that orchestrate urinary bladder development should be activated in adult to induce urinary bladder regrowth. In most cases, we focus our attention and research efforts on selected signaling pathways, and in turn, we risk overlooking connections between them. In this study, for the first time, we applied genome wide microarray analysis of gene expression to obtain a broad view of signaling pathways influenced by mesenchymal stromal cells. We aimed to determine the complexity of the healing response by implanted stem cells. Based on the obtained results, in the next step, we focused on the analysis of the Hedgehog signaling pathway that is one of the most important pathways for normal urinary bladder development.

\section{Methods \\ Study design}

Rat urinary bladders were reconstructed with bladder acellular matrix (BAM) $(n=52)$ or BAM seeded with adipose tissue-derived mesenchymal stromal cells (ASCs) $(n=52)$. The process of bladder healing was analyzed at 7 , 30,90 , and 180 days postoperatively using macroscopic, histologic, and molecular techniques. Gene expression was analyzed by microarrays and confirmed by real-time PCR. Experiment workflow is presented in Fig. 1.

\section{Rats}

Male Wistar rats from the Mossakowski Medical Research Center, Polish Academy of Sciences, were used as donors of adipose tissue for stem cells isolation and urinary bladders for acellular matrices preparation as well as an experimental model of urinary bladder augmentation. The study was carried out in strict accordance with recommendations from the Guide for the Care and Use of Laboratory Animals of the National Institutes of Health [13]. The protocol was approved by the Nicolaus Copernicus University Ethics Committee (no. 46/2012).

\section{Adipose tissue-derived mesenchymal stromal cells isolation and culture}

Adipose tissue-derived mesenchymal stromal cells (ASCs) were isolated from the retroperitoneal adipose tissue harvested from 52 male Wistar rats and cultured as previously described [12]. Briefly, an adipose tissue was digested in collagenase $\mathrm{P}$ (Roche Diagnostics GmbH, Switzerland) in Hank's Balanced Salt Solution (HBSS; Pan-Biotech, Germany) $(1 \mathrm{mg} / \mathrm{ml})$ supplemented with calcium chloride ( $5 \mathrm{mM}$, Sigma-Aldrich, Germany) and HEPES Buffer (PAA, Austria) at a concentration of $1 \mathrm{ml}$ enzyme solution $/ 1 \mathrm{~g}$ of tissue for $10 \mathrm{~min}$ at $37^{\circ} \mathrm{C}$. Isolated cells were cultured in Dulbecco's modified Eagle's medium/Ham's F12 (DMEM/Ham's F12) (HyClone, USA) supplemented with $10 \%$ fetal bovine serum (FBS) (Sigma-Aldrich, Germany), basic fibroblast growth factor (b-FGF) (10 ng/ml, Gibco, USA), penicillin/streptomycin $(100 \mathrm{U} / \mathrm{ml} / 100 \mu \mathrm{g} / \mathrm{ml}$, HyClone, USA), and amphotericin B $(5 \mu \mathrm{g} / \mathrm{ml}$, Corning, USA $)$ at $37^{\circ} \mathrm{C}$ in $5 \% \mathrm{CO}_{2}$ atmosphere and $95 \%$ humidity until the third passage.

\section{Adipose tissue-derived mesenchymal stromal cell immunophenotype and multipotency}

The expression of ASCs surface markers was analyzed to confirm their immunophenotype. For this purpose, 


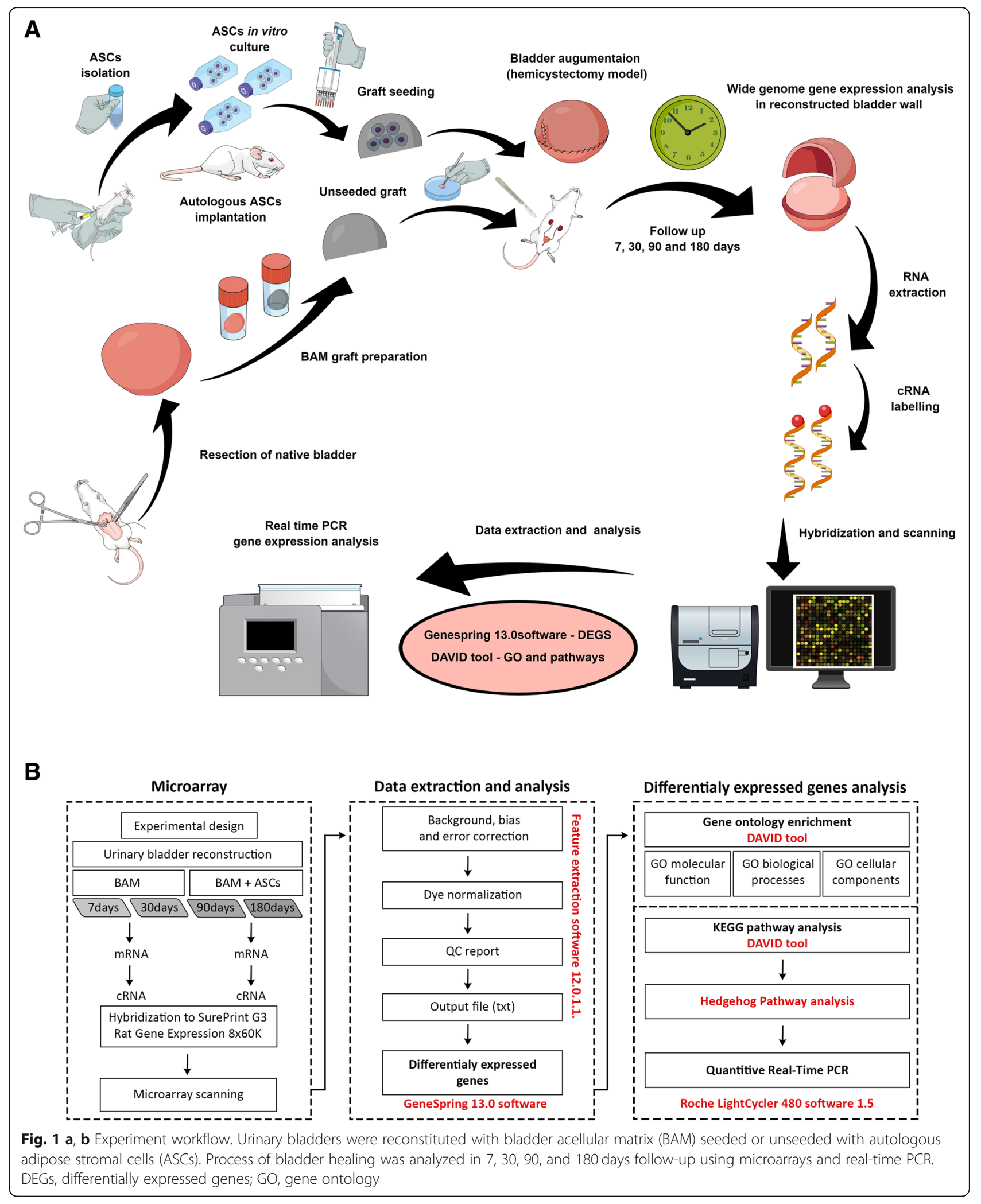

ASCs were incubated with fluorescein isothiocyanate (FITC)- or phycoerythrin (PE)-conjugated monoclonal antibodies against CD11b, CD29, CD31, CD34, CD44,
CD45, and CD90 (BD Biosciences, USA, Santa Cruz Biotechnology, USA) for $30 \mathrm{~min}$ at $4{ }^{\circ} \mathrm{C}$ in dark. FITC- or PE-conjugated IgG1, IgG2a, IgM, and IgA were used as 
isotype controls (BD Biosciences, USA). Cell surface marker expression was analyzed on BD FACSCanto II using BD FACSDiva ${ }^{\mathrm{Tn}}$ Software (BD Biosciences, USA). The multipotential character of ASCs was confirmed by their ability to differentiate into adipogenic, chondrogenic, and osteogenic lineages in appropriate media (Mesenchymal Adipogenesis Kit, Merck Millipore, USA; StemPro $^{\text {tw }}$ Chondrogenesis Differentiation Kit, Life Technologies, USA; StemPro ${ }^{\mathrm{mm}}$ Osteogenesis Differentiation Kit, Life Technologies, USA) following the manufacturer's instruction. ASCs cultured in the standard medium were used as a control. Adipogenesis, chondrogenesis, and osteogenesis were confirmed by Oil Red $\mathrm{O}$ (Merck Millipore, USA), Alcian Blue (Sigma-Aldrich, Germany), and Alizarin Red (Merck Millipore, USA) stainings, respectively.

\section{Bladder acellular matrix}

Urinary bladders were harvested from 113 male Wistar rats and washed in sterile phosphate-buffered saline (PBS; Pan-Biotech, Germany) supplemented with penicillin/streptomycin $\quad(100 \mathrm{U} / \mathrm{ml} / 100 \mu \mathrm{g} / \mathrm{ml}$, HyClone, USA) and amphotericin B ( $5 \mu \mathrm{g} / \mathrm{ml}$, Corning, USA). Subsequently, the urinary bladders were longitudinally sectioned and then submucosa and urothelium layers were removed by manual scraping. Next, the urinary bladder tissues were frozen $\left(-80^{\circ} \mathrm{C}\right)$ and thawed $\left(37^{\circ} \mathrm{C}\right)$ three times in 5-mM ethylenediaminetetraacetic acid (EDTA; Sigma-Aldrich, Germany) and 10-mM Tris $\mathrm{HCl}$ (Life Technologies, USA) solution and then placed in isopropanol ( $\geq 99.5 \%$, Sigma-Aldrich, Germany) followed by overnight incubation at room temperature. After that, the urinary bladder tissues were transferred to $0.5 \%$ Triton X-100 (Sigma-Aldrich, Germany) and $26.5 \mathrm{mM}$ ammonium hydroxide (Honeywell, USA) solution for 14 days with fresh solution change every 3-4 days. Subsequently, the urinary bladder tissues were washed overnight in Hank's balanced salt solution (HBSS; Pan-Biotech, Germany) supplemented with benzonase nuclease $\left(2 \mathrm{U} / \mathrm{mL}\right.$, Sigma-Aldrich, Germany) at $37^{\circ} \mathrm{C}$. Finally, acellular matrices were thoroughly washed with sterile double-distilled water and stored in 70\% ethanol (Avantor Performance Materials, Poland) prior to use. The bladder matrices $(n=3)$ were evaluated by scanning electron microscopy (SEM; Auriga 60 scanning microscope, Zeiss, Germany) to confirm their acellularity.

\section{Graft preparation}

ASCs from the third passage were seeded on BAMs in a density of $10 \times 10^{6} / \mathrm{cm}^{2}$ and cultured for 7 days in Dulbecco's modified Eagle's medium/Ham's F12 (DMEM/ Ham's F12) (HyClone, USA) supplemented with 10\% fetal bovine serum (FBS) (Sigma-Aldrich, Germany), basic fibroblast growth factor (bFGF) $(10 \mathrm{ng} / \mathrm{ml}$, Gibco, USA), penicillin/streptomycin $(100 \mathrm{U} / \mathrm{ml} / 100 \mu \mathrm{g} / \mathrm{ml}$, HyClone, USA), and amphotericin B $(5 \mu \mathrm{g} / \mathrm{ml}$, Corning, USA) at $37^{\circ} \mathrm{C}$ in $5 \% \mathrm{CO}_{2}$ atmosphere and $95 \%$ humidity. Cell morphology and the distribution of the cells into the scaffold were analyzed by SEM $(n=3)$.

\section{Urinary bladder augmentation}

One hundred and four syngeneic male Wistar rats weighing between 250 and $300 \mathrm{~g}$ were randomly divided into eight equal groups $(n=13)$. Rats were anesthetized with sodium pentobarbital $(15 \mathrm{mg} / \mathrm{kg}$, i.p., Biowet, Poland) and lidocaine $(20 \mathrm{mg} / \mathrm{kg}$, i.m., Polfa, Poland) before undergoing hemicystectomy and bladder reconstruction with a graft of approximately $1 \mathrm{~cm}^{2}$ in size. Urinary bladders were reconstructed with BAM (first, second, third, and fourth groups) or BAM seeded with ASCs (fifth, sixth, seventh, and eighth groups). The animals were sacrificed after 7 (first and fifth groups), 30 (second and sixth groups), 90 (third and seventh groups), and 180 (fourth and eighth groups) days. The reconstructed bladders were harvested for macroscopic, histological, and molecular analyses.

\section{Histological stainings}

Two independent pathologists analyzed the regeneration of urothelium, smooth muscles, and inflammatory response in urinary bladder tissue sections stained routinely with H\&E and Masson's trichrome. The analysis was performed for three biological and three technical samples from each group.

\section{Microarrays}

Reconstructed bladder walls were separated from native bladder tissues, placed in RNAlater solution (Thermo Fisher Scientific, USA), and stored at $-80^{\circ} \mathrm{C}$. Total RNA was isolated from 10 bladders, from each group using a High Pure RNA Tissue Kit (Roche Diagnostics, Switzerland) according to the manufacturer's instructions. Quantity and purity of RNA were evaluated using a NanoDrop (Thermo Scientific, USA) and the integrity of the RNA was determined using a RNA 6000 Nano Kit on the Agilent 2100 BioAnalyzer (Agilent Technologies, USA). Only RNA samples with a RNA Integrity Number (RIN) above 7.0 were used for further analysis. Eight samples from each group (biological replicates) were chosen and subjected to microarray experimentation with the use of a SurePrint G3 Rat Gene Expression 8x60K Kit (Agilent Technologies, USA) in accordance with the One-Color Microarray-Based Gene Expression Analysis Protocol (Agilent Technologies, USA). Slides were scanned on a NimbleGen MS 200 Microarray Scanner (Roche, Switzerland), and data was extracted from images using Agilent Feature Extraction software 
12.0.1.1. (Agilent Technologies, USA). Gene expression data analysis was performed with the use of GeneSpring 13.0 software (Agilent Technologies, USA). Significant differences in gene expression were determined by a two-way ANOVA test using Benjamini Hochberg correction. Hierarchical clustering was performed to show gene expression changes in all groups over time. Differentially expressed genes (DEGs) with fold change greater than 1.5 and statistical significance $p<0.01$ were subjected to DAVID 6.8; Database for Annotation, Visualization and Integrated Discovery Classification System [14, 15]. for further analysis. Pathway analysis was performed using WikiPathways and the KEGG Pathway Database. List of DEGs uploaded to DAVID allowed for GO analysis to be performed, including functional categories: biological processes, molecular function, and cellular components. Only, GO with $p<0.05$ were considered. The experimental data were prepared according to MIAME 2.0 standards and deposited in GEO, NCBI.

\section{Quantitative real-time PCR}

Expression of Hedgehog pathway-related genes was quantified using real-time PCR. cDNA was synthesized from $500 \mathrm{ng}$ of total RNA from ten bladders of each group according to the manufacturer's protocol with the use of Transcriptor First Strand cDNA Synthesis Kit (Roche Diagnostics, Switzerland). RealTime ready Custom Panel 96 (Roche Diagnostics, Switzerland) was used for quantitative real-time PCR in accordance with the manufacturer's protocol. The primer and probe sequences were presented in Additional file 1: Table S1. The LightCycler 480 cycling parameters were denaturation in $95^{\circ} \mathrm{C}$ for 10 min followed by 45 cycles of $95^{\circ} \mathrm{C}$ for $10 \mathrm{~s}, 60^{\circ} \mathrm{C}$ for $30 \mathrm{~s}$, and $72{ }^{\circ} \mathrm{C}$ for $1 \mathrm{~s}$ and after amplification a $40^{\circ} \mathrm{C}$ cooling period for $30 \mathrm{~s}$. The LightCycler 480 software 1.5 (Roche Diagnostics, Switzerland) was used to perform advanced relative quantification analysis. Statistical analysis of gene expressions was performed using IBM SPSS Statistics Software (Poland). All data are presented as a mean \pm SD. Two group comparisons were done with Student's $T$ test. A value of $p<0.05$ was taken as statistically significant.

\section{Data availability}

The microarray gene expression data have been deposited at the NCBI Gene Expression Omnibus. The data are under accession number GEO: GSE103572.

\section{Results}

\section{Analysis of cells and grafts}

ASCs had a typical MSCs immunophenotype that was characterized by high expression of CD29 $(100.00 \% \pm 0.00)$, CD44 $(92.07 \% \pm 6.00)$ and CD90 $(90.83 \% \pm 4.53)$ markers and low expression of CD11b $(0.77 \% \pm 0.31), \quad \mathrm{CD} 31$
$(1.00 \% \pm 0.33)$, and CD45 $(1.14 \% \pm 0.45)$ markers (Additional file 5: Figure S1A). ASCs had multipotent differentiation properties; they were able to differentiate into adipogenic, chondrogenic, and osteogenic lineages (Additional file 5: Figure S1B-D). ASCs cultured in standard medium remained undifferentiated (Additional file 5: Figure S1E-G). Prepared bladder acellular matrices (BAM) were free from cells and their debris (Additional file 5: Figure $\mathrm{S} 1 \mathrm{H})$. ASCs seeded into BAM had proper morphology. They formed a homogeneous layer by developing the networks of cell-cell and cell-biomaterial connections. Only single ASCs had spherical shape (Additional file 5: Figure S1IJ).

\section{Macroscopic and histological analysis of reconstructed bladders}

Macroscopically, both BAM seeded with or without ASCs integrated positively with the host's native bladder tissues (Fig. 2). At days 7 and 30, the differences between urinary bladders reconstructed with BAM seeded with or without ASCs (first vs fifth and second vs sixth groups) were unnoticeable. However, at days 90 and 180 days, the bladders reconstructed with BAM seeded with ASCs (seventh and eighth groups) showed enhanced angiogenesis and less visible graft shrinkage compared to bladders reconstructed with only BAM (third and fourth groups) (Fig. 2). Higher frequency of complications including, stenosis between the graft and native bladder tissue, stone formation, and peritoneal adhesions was observed in bladders reconstructed with BAM only compared to BAM seeded with ASCs.

Histologically, there were no significant differences in urothelium regeneration between the bladders reconstructed with BAM only compared to BAM seeded with ASCs. The luminal surface of the graft was completely covered by a multilayered (five cell layers) urothelium within 7 days. In certain cases, the thickness of urothelium was decreased $(<4$ cell layer). The morphology of the epithelial tissue was normal with an exception of one case, in which inflamed urothelium was observed (Additional file 6: Figure S2A-C, J). ASCs enhanced smooth muscle regeneration. The differences in smooth muscle regeneration between the bladders reconstructed with BAM only compared to the bladders reconstructed using BAM seeded with ASCs were observed as early as at 7 days post reconstruction. In the bladders reconstructed only with BAM (first group), the regeneration of the smooth muscle tissue at day 7 of follow-up was not observed, while in the bladders reconstructed using BAM seeded with ASCs (fifth group), the smooth muscle tissue started to regenerate forming single chaotically distributed smooth muscle fibers. The content of smooth muscle tissue increased gradually at days 30 and 90 of follow-up, but the smooth muscle fibers arrangement was irregular. Completely regenerated smooth muscle layer with well-organized smooth muscle fibers was observed 

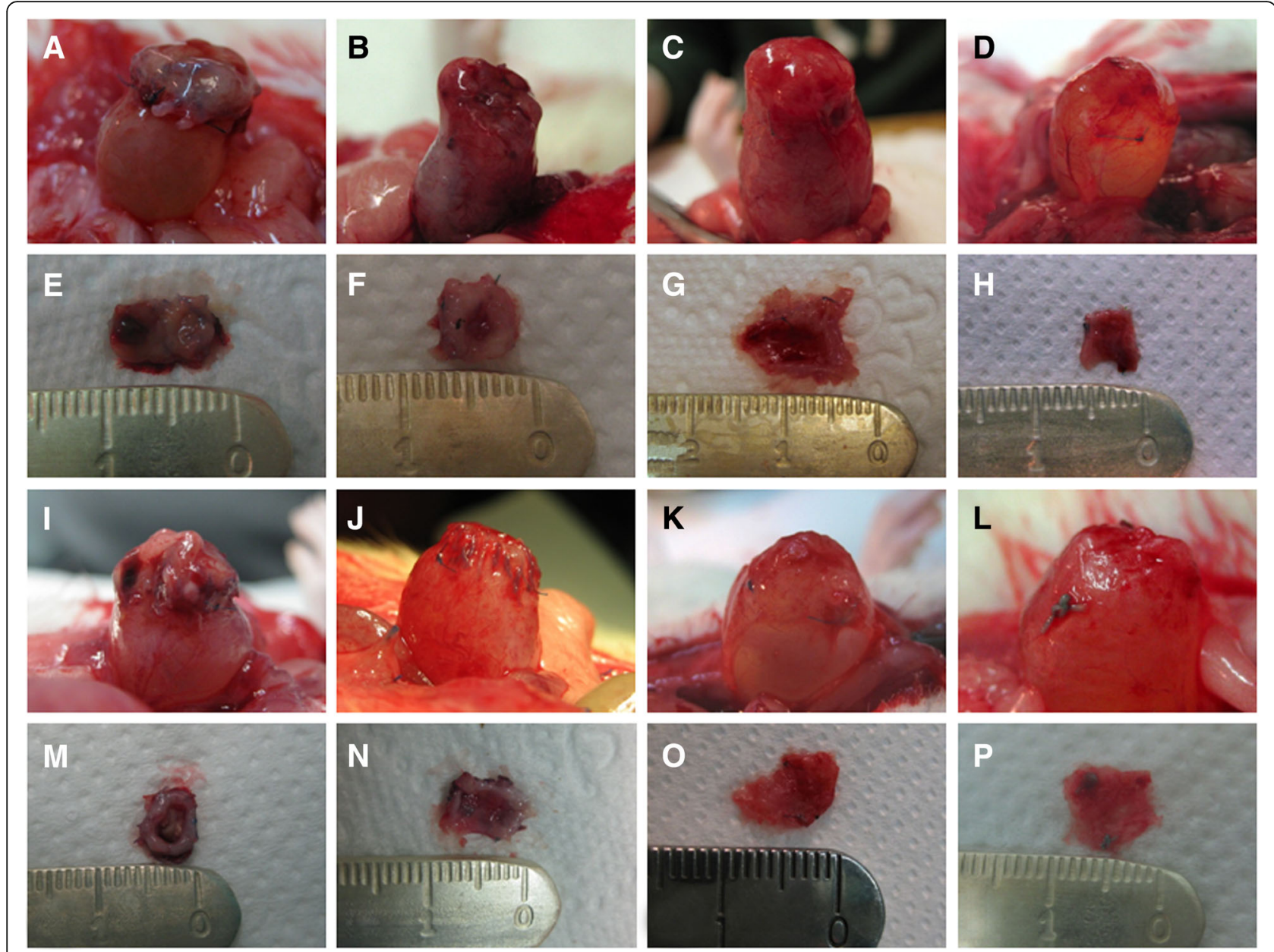

Fig. 2 Macroscopic analysis of urinary bladders reconstructed with BAM only $(\mathbf{a}-\mathbf{d})$ or BAM seeded with ASCs (i-I) at $7(\mathbf{a}, \mathbf{i}), 30(\mathbf{b}, \mathbf{j}), 90(\mathbf{c}, \mathbf{k})$, and $180(\mathbf{d}, \mathbf{l})$ days after the surgery. Bladder walls reconstructed with BAM only $(\mathbf{e}-\mathbf{h})$ or BAM seeded with ASCs $(\mathbf{m}-\mathbf{p})$ separated from native bladder tissue at $7(\mathbf{e}, \mathbf{m}), 30(\mathbf{f}, \mathbf{n}), 90(\mathbf{g}, \mathbf{o})$, and $180(\mathbf{h}, \mathbf{p})$ days after the surgery. Reconstructed bladder wall was macroscopically indistinguishable from native bladder tissue in the bladders augmented with ASC-seeded BAM after 90 and 180 days following the reconstruction (k, I). Graft shrinkage was observed in bladders augmented with BAM only; the process progressed with time and was the most intensive at 180 days follow-up (h). The images are representative from 13 experiments performed per each group $(n=104)$

only in bladders reconstructed using BAM seeded with ASCs in 180 days post-reconstruction (eighth group)(Additional file 6: Figure S2D-F, K). An intense inflammatory response was observed in bladders reconstructed using BAM seeded with or without ASCs after day 7 of follow-up. The intensity of inflammation decreased with time (Additional file 6: Figure S2G-I, L).

\section{Hierarchical clustering of differentially expressed genes}

Significant gene expression changes between the bladders reconstructed using BAM seeded with or without ASCs at 7, 30, 90, and 180 days postoperatively were observed (Fig. 3a, b). The hierarchical clustering showed distinguishable gene expression profiling between the reconstructed bladders (Fig. 4). The hierarchical clustering performed for all experimental groups divided differentially expressed genes (DEGs) in two main clusters: short
7 and 30, and long 90 and 180 days follow-up. The gene expression profile in bladders reconstructed using BAM seeded with ASCs at day 7 was comparable to the profile observed in the bladders reconstructed using BAM only at day 30 of follow-up. This differed from the gene expression profile observed in the bladders reconstructed using BAM seeded with ASCs at 30 days follow-up. In contrast, the gene expression profile of bladders reconstructed using BAM seeded with or without ASCs at 180 days follow-up was comparable but differed from the gene expression profile observed in the bladders reconstructed using BAM seeded with ASCs or BAM only at 90 days follow-up (Fig. 3a). The hierarchical clustering performed separately for different observation times revealed distinguishable gene expression profiling between bladders reconstructed with BAM seeded with ASCs and BAM only (Fig. 4). 


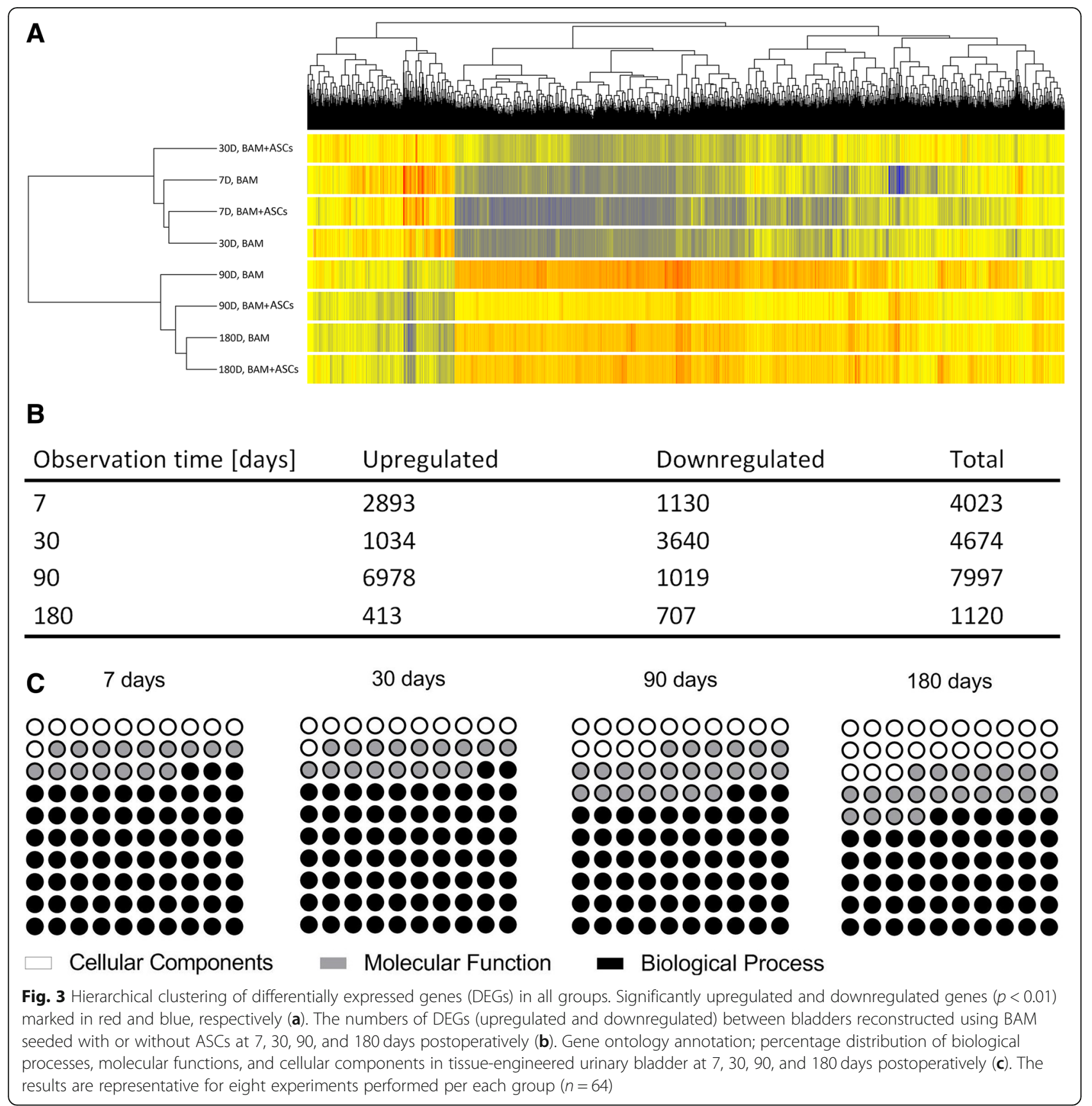

Enriched pathways analysis

Involvement of DEGs in Wiki and KEGG pathways in bladder healing at 7, 30, 90, and 180 days postoperatively was presented in Additional file 2: Table S2 and Additional file 3: Table S3. The most crucial differentially activated signaling pathways for bladders reconstructed using BAM seeded with or without ASCs are presented in Fig. 5. Of particular interest are pathways being a key regulator of embryonic development, involved in multiple processes including cell fate determination, tissue patterning, and morphogenesis as well as regulation of adult stem cell renewal, maintenance of homeostasis, and regeneration of adult tissues such us Hedgehog, TGF- $\beta$, Jak-STAT, PI3-Akt, and Hippo signaling pathways. Another very interesting observation is strong modulation of immune response by ASCs manifested by changes in numerous pathways including cytokinecytokine interaction, allograft rejection, leukocyte transendothelial migration, and chemokine signaling (Fig. 5, Additional file 2: Table S2 and Additional file 3: Table S3). 
A

7D

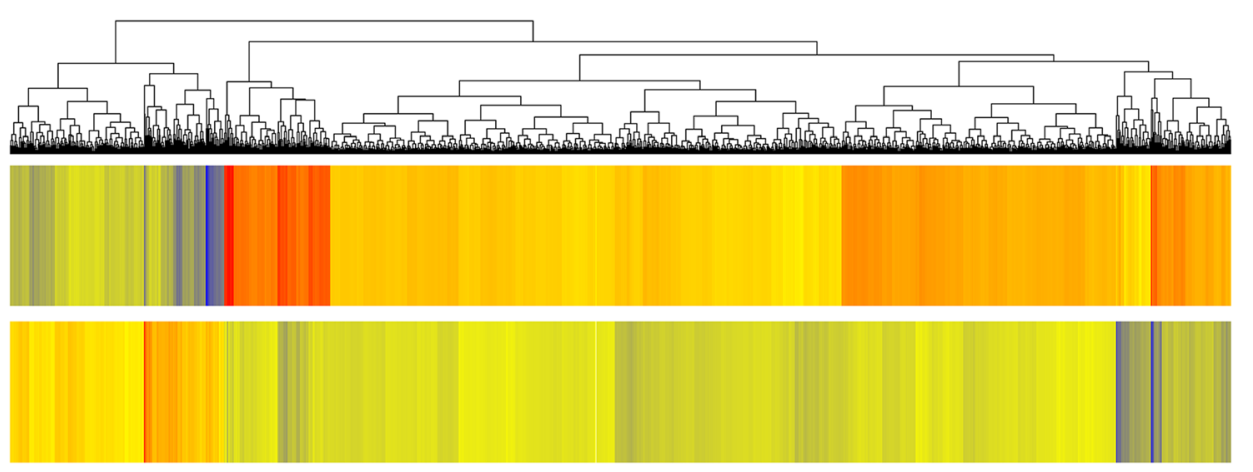

BAM

BAM + ASCS

B

30D
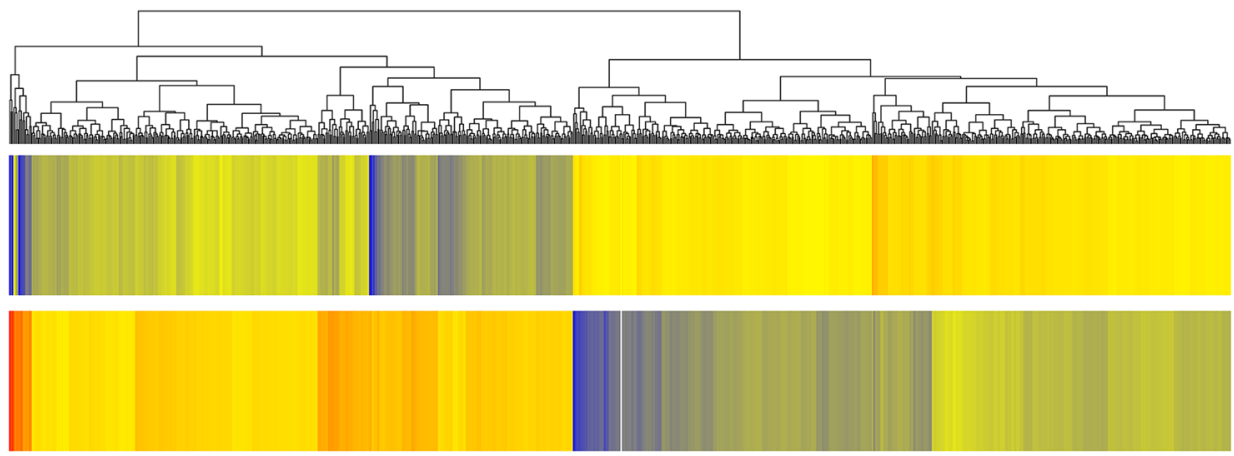

BAM

BAM + ASCs

C

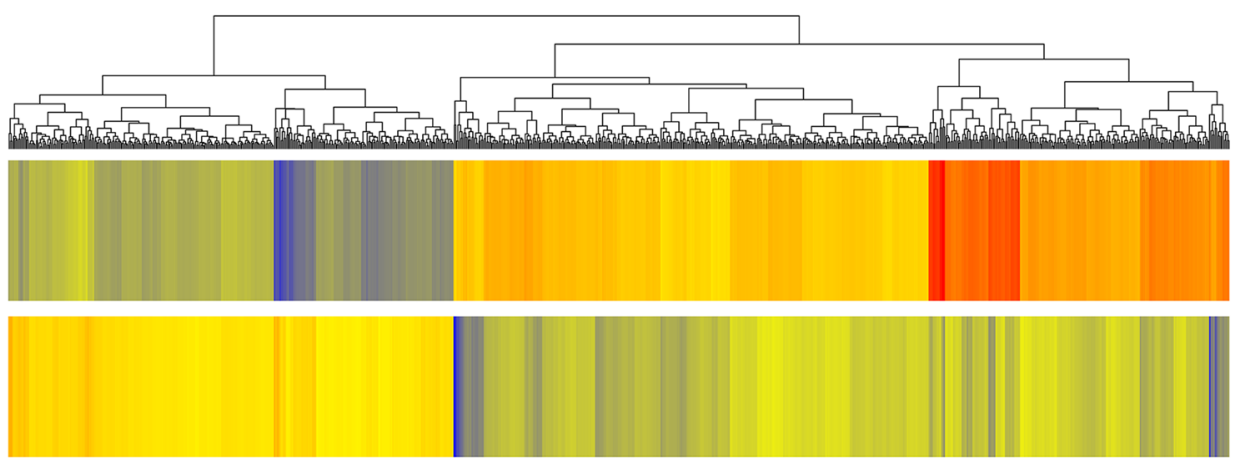

BAM

BAM + ASCs

D

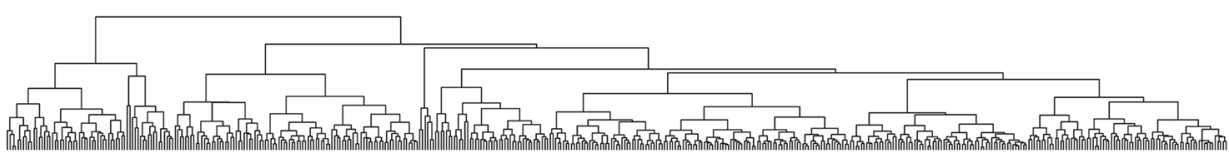

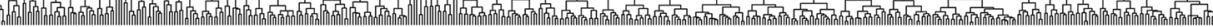

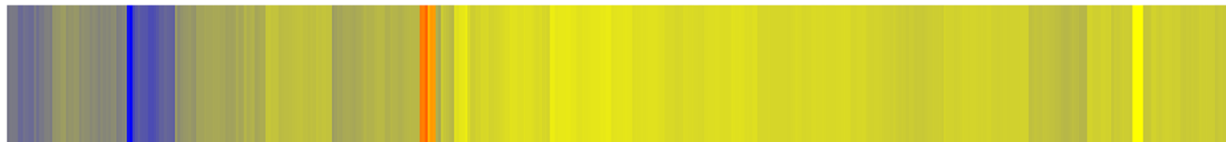

BAM

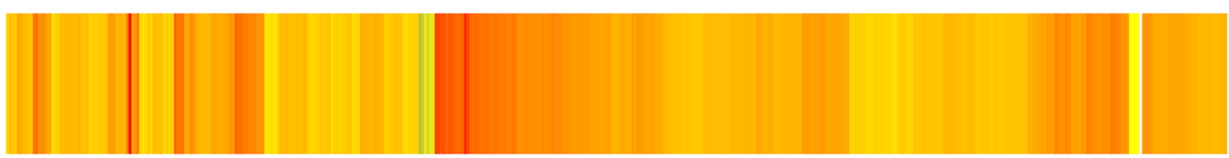

BAM + ASCs

DOWN

Fig. 4 Hierarchical clustering of differentially expressed genes (DEGs) between bladders reconstructed using BAM seeded with ASCs vs BAM only in different time point, at 7 (a), 30 (b), 90 (c), and 180 (d) days postoperatively. Significantly upregulated and downregulated genes $(p<0.01)$ are marked in red and blue, respectively. The results are representative for eight experiments performed per each group $(n=64)$ 


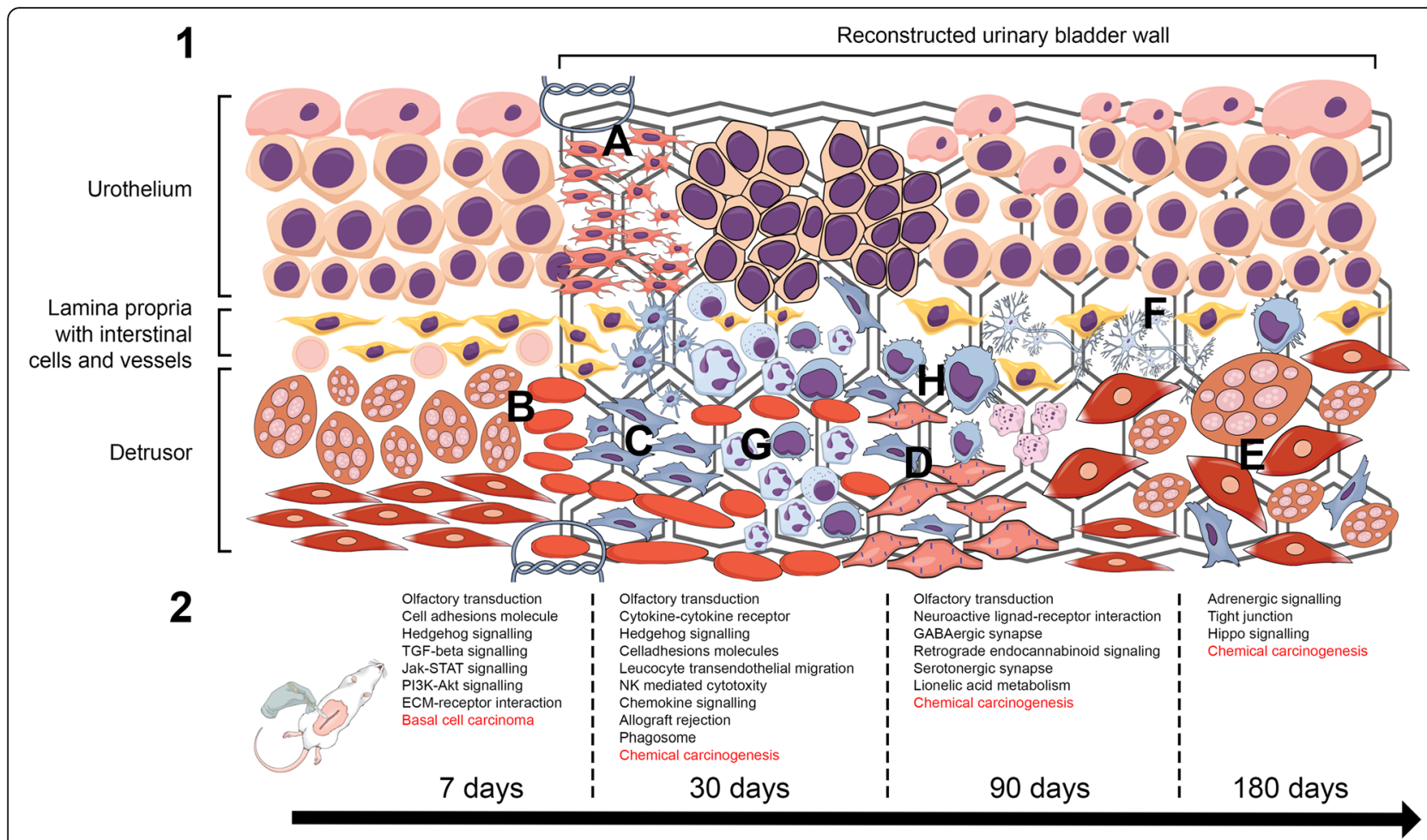

Fig. 5 The healing process of tissue-engineered urinary bladder. 1. Shortly after bladder reconstruction with an in vitro constructed graft, urothelial (a) and detrusor cells (b) dedifferentiate into actively proliferating cells that migrate into the graft, populate it, and restore the neobladder wall. Simultaneously, as a part of healing process, fibroblasts begin to proliferate and form initial scar tissue (c) to limit the injury site. Even though precursors of smooth muscle cells elongate within the graft (d), their initial layered architecture is disrupted. The regenerated detrusors (e) characterizes with irregular smooth muscle bundle arrangement. The intestinal cells play an unknown role during bladder wall regeneration. They might however regulate restoration of the neuronal compartment of the bladder wall by interacting with regenerating neurons $(f)$ at different time points after reconstruction. The inflammatory response comprises an initial acute phase and a subsequent chronic phase. The acute phase lasts from hours to days and is mediated mainly by neutrophilic reactions (g). Monocytes are then called into the site, and these differentiate into macrophages that are primary cells maintaining the chronic phase (h). 2. Differentially expressed pathways in bladders reconstructed with BAM seeded with or without ASCs at 7, 30, 90, and 180 days postoperatively. The most crucial signaling pathways for each period are provided. Active carcinogenic pathways are marked with red

\section{Enriched gene ontology analysis}

Gene ontology (GO) functional enrichment analysis performed for DEGs allowed for identification of biological processes, molecular functions, and cellular components significant for urinary bladder healing at 7, 30, 90, and 180 days postoperatively (Fig. 3c). Selected ontologies categorized into cellular and intercellular events, morphogenesis, angiogenesis, epithelium, muscles and nerve regeneration, extracellular matrix remodeling, signal transduction, inflammatory response, and wound healing are presented in Additional file 4: Table S4.

\section{Hedgehog signaling pathway gene expression analysis: microarray data}

Numerous differentially expressed genes and pathways between the bladders reconstructed with ASCs seeded and unseeded BAM were identified. The Hedgehog signaling pathway was found to be significantly enriched after 7 and 30 days after the bladder reconstruction $(p<0.05)$. Numerous Hedgehog pathway genes were upregulated in the bladders reconstructed with ASCs (Fig. 6). These results clearly show that mesenchymal stromal cells activate the Hedgehog signaling in reconstructed bladder. Increased expression of Wnt and bone morphogenic protein (Bmp) genes together with Hedgehog genes suggest their synergistic role in urinary bladder regeneration. Surprisingly, the significant differences in expression of Hh, Wnt, and Bmp genes between the bladders reconstructed with or without mesenchymal stromal cells were observed only in short 7 and 30 days observation times, and then diminished.

\section{Hedgehog signaling pathway gene expression analysis: real-time PCR data}

Real-time PCR analysis confirmed upregulation of genes involved in the Hedgehog signaling pathways (Figs. 6 and 7). At 7 and 30 days after the bladder reconstruction, increased expression of genes coding Sonic Hedgehog (Shh) and Indian Hedgehog (Ihh) ligand and their Patched 1 


\begin{tabular}{|c|c|c|c|c|c|c|c|c|c|c|c|}
\hline \multirow{2}{*}{$\frac{A}{\text { Gene symbol }}$} & \multicolumn{5}{|c|}{ MICROARRAY } & \multicolumn{6}{|c|}{ REAL-TIME PCR } \\
\hline & Gene ID & $7 \mathrm{~d}$ & $30 d$ & $90 \mathrm{~d}$ & $180 \mathrm{~d}$ & Gene symbol & Gene ID & $7 d$ & $30 \mathrm{~d}$ & $90 \mathrm{~d}$ & $180 \mathrm{~d}$ \\
\hline Bmp2 & 29373 & $\Delta$ & 0 & $\Delta$ & 0 & Bmp2 & 29373 & $\Delta$ & 0 & 0 & 0 \\
\hline Bmp4 & 25296 & $\boldsymbol{\Delta}$ & ○ & & 0 & Bmp4 & 25296 & $\Delta$ & - & 0 & 0 \\
\hline Dhh & 84380 & $\nabla$ & $\Delta$ & & - & Dhh & 84380 & $\nabla$ & $\Delta$ & 0 & \\
\hline Gli1 & 140589 & $\Delta$ & - & - & & Gli1 & 140589 & $\boldsymbol{\Delta}$ & - & - & \\
\hline Ihh & 84399 & $\Delta$ & $\Delta$ & $\Delta$ & $\nabla$ & Ihh & 84399 & $\Delta$ & $\Delta$ & 0 & $\nabla$ \\
\hline Lrp2 & 29216 & - & $\Delta$ & $\nabla$ & - & Lrp2 & 29216 & - & $\overline{0}$ & 0 & 0 \\
\hline Ptch1 & 89830 & $\Delta$ & $\Delta$ & 0 & & Ptch1 & 89830 & & & & \\
\hline Rab23 & 367242 & $\nabla$ & $\mathbf{\Delta}$ & $\nabla$ & 0 & Rab23 & 367242 & 0 & 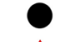 & 0 & \\
\hline Shh & 29499 & 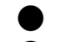 & $\Delta$ & 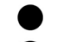 & & Shh & 29499 & $\Delta$ & $\Delta$ & 0 & \\
\hline Smo & 25273 & - & - & - & - & Smo & 25273 & - & $\Delta$ & - & - \\
\hline Wnt1 & 24881 & $\nabla$ & - & $\nabla$ & $\Delta$ & Wnt1 & 24881 & 0 & - & 0 & \\
\hline Wnt10a & 316527 & $\Delta$ & $\Delta$ & & & Wnt10a & 316527 & $\Delta$ & $\Delta$ & 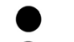 & \\
\hline Wnt10b & 315294 & - & $\Delta$ & $\nabla$ & 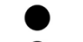 & Wnt10b & 315294 & - & - & 0 & \\
\hline Wnt16 & 500047 & $\Delta$ & $\Delta$ & $\nabla$ & & Wnt16 & 500047 & - & - & - & \\
\hline Wnt2 & 114487 & $\boldsymbol{\Delta}$ & - & 0 & 0 & Wnt2 & 114487 & $\boldsymbol{\Delta}$ & $\Delta$ & ○ & 0 \\
\hline Wnt2b & 116466 & $\Delta$ & - & 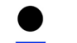 & - & Wnt2b & 116466 & $\Delta$ & $\Delta$ & $\Delta$ & \\
\hline Wnt3 & 24882 & 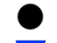 & $\Delta$ & & & Wnt3 & 24882 & 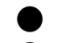 & - & 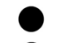 & \\
\hline Wnt3a & 303181 & $\nabla$ & 0 & • & 0 & Wnt3a & 303181 & ○ & - & - & \\
\hline Wnt4 & 84426 & - & $\Delta$ & $\Delta$ & ? & Wnt4 & 84426 & - & $\Delta$ & $\Delta$ & \\
\hline Wnt5a & 64566 & 0 & $\Delta$ & - & & Wnt5a & 64566 & 0 & $\Delta$ & $\Delta$ & \\
\hline Wnt7b & 315196 & 은 & $\Delta$ & 인 & 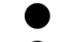 & Wnt7b & 315196 & ○ & 0 & ○ & \\
\hline Wnt8b & 293990 & $\nabla$ & $\Delta$ & $\nabla$ & & Wnt8b & 293990 & - & - & - & ? \\
\hline Wnt9a & 287357 & $\nabla$ & $\Delta$ & • & 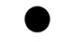 & Wnt9a & 287357 & 0 & - & 0 & 0 \\
\hline Zic2 & 287357 & $\nabla$ & $\nabla$ & $\Delta$ & $\Delta$ & Zic2 & 287357 & 0 & $\bullet$ & $\bullet$ & - \\
\hline Expression: & $\Delta$ upreg & & & downre & ulated & unchanged & & & & & \\
\hline \multicolumn{12}{|c|}{$\begin{array}{l}\text { Fig. } 6 \text { Hedgehog-related gene expression analysis in urinary bladders reconstructed with bladder acellular matrix (BAM) seeded with adipose } \\
\text { stromal cells (ASCs) compared to bladders reconstructed with BAM only in 7, 30, 90, and } 180 \text { days following the surgery. Analysis by microarrays } \\
\text { (a): genes with } p<0.01 \text { and fold change }>1.5 \text { were defined as differentially expressed; and real-time PCR (b): genes with } p<0.05 \text { were defined as } \\
\text { differentially expressed. Genes with unchanged, upregulated and downregulated expression are presented }\end{array}$} \\
\hline
\end{tabular}

(Ptch1) receptor was observed in the bladders reconstructed with mesenchymal stromal cells. This resulted in increased expression of Smoothened (Smo) gene and consequently increased expression of Shh target genes including bone morphogenetic proteins (Bmp) and Wnt. Bmp2 and Bmp4 genes were upregulated in the bladders reconstructed with stem cells only in the early stage of healing (7 days follow-up). While upregulated expression of Wnt family genes including Wnt2, Wnt2b, Wnt4, Wnt5a, and Wnt10a in later stages of healing (30 or even 90 days follow-up). Our findings, summarized schematically in Fig. 8, reveal an essential contribution of $\mathrm{Hh}$, Wnt, and Bmp signals during regeneration of tissue-engineered urinary bladder.

\section{Discussion}

The ultimate quality of reconstructed bladder wall is dependent from restoration of the urothelium and detrusor cytoarchitecture [16, 17]. The final regenerative effect is predominantly linked to sequenced proliferation and differentiation of activated bladder urothelial and smooth muscle progenitor cells. The self-regenerating capability is not however enough to rebuild the primary bladder wall structure. Therefore, boosting endogenous regenerative potency is necessary [5]. Stem cells stimulate regeneration of tissue-engineered urinary bladder but the molecular basis of this process remains unknown until now.

To our knowledge, it is the first study that provided unequivocal evidence that implanted ASCs changed the molecular pattern of healing in bladders reconstructed with a tissue engineering approach. Applied microarray analysis exposed a significant host response on stimuli delivered by ASCs. A total of 4023, 4674, 7997, and 1120 differentially expressed genes (DEGs) between the bladders augmented with BAM seeded with or without ASCs were identified at 7, 30, 90, and 180 days postoperatively. Detailed analysis of DEGs allows to determine several molecular pathways that can be associated with the regeneration process, among them the pathways being a key regulator of embryonic development, involved in multiple processes including cell fate determination, 


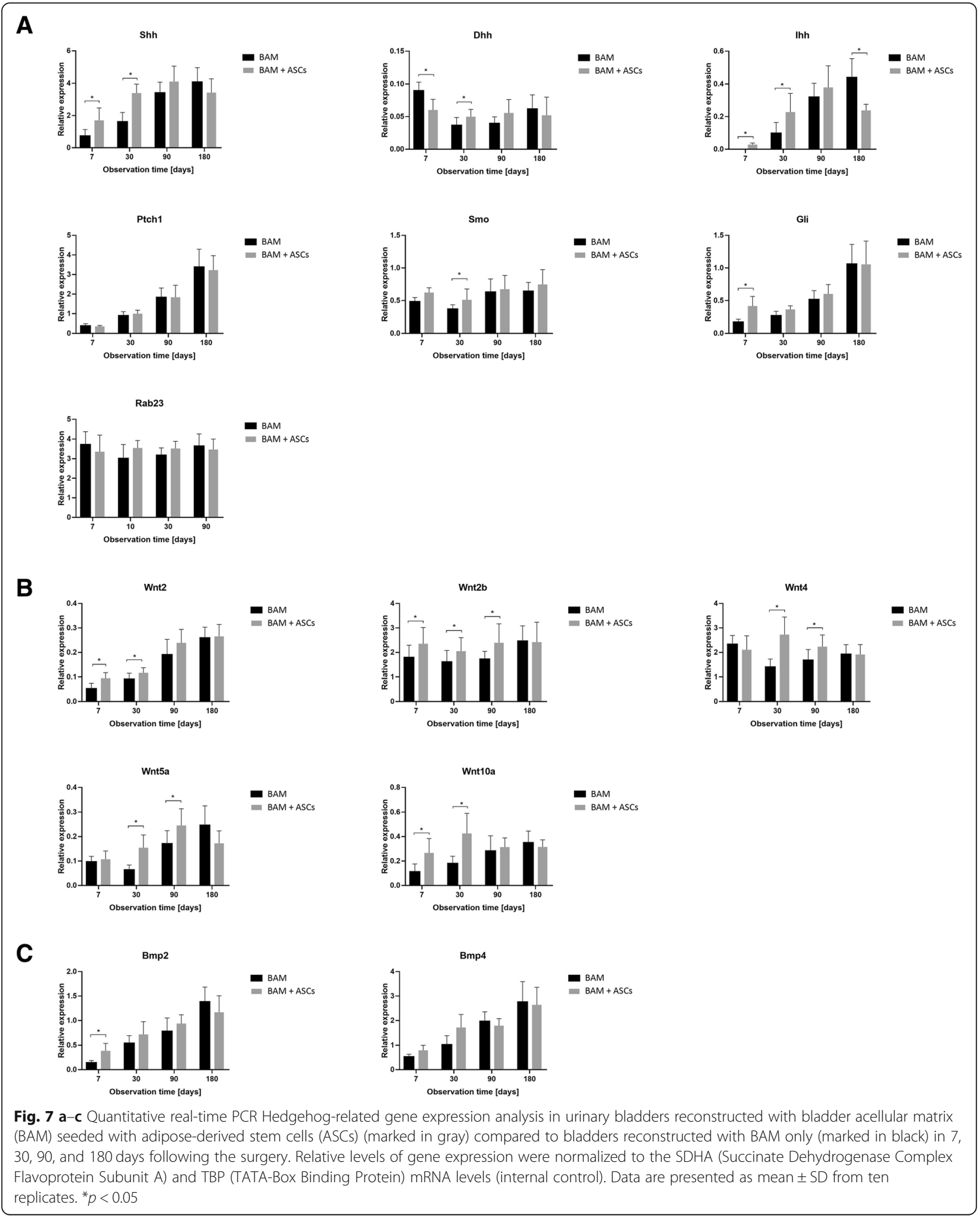

tissue patterning, and morphogenesis as well as regulation of adult stem cell renewal, maintenance of homeostasis, and regeneration of adult tissues, such as TGF- $\beta$,
Jak-STAT, PI3-Akt, Hippo, and Hedgehog signaling pathways. Observed differences between cellular and acellular grafts justify using ASCs as agents that create 


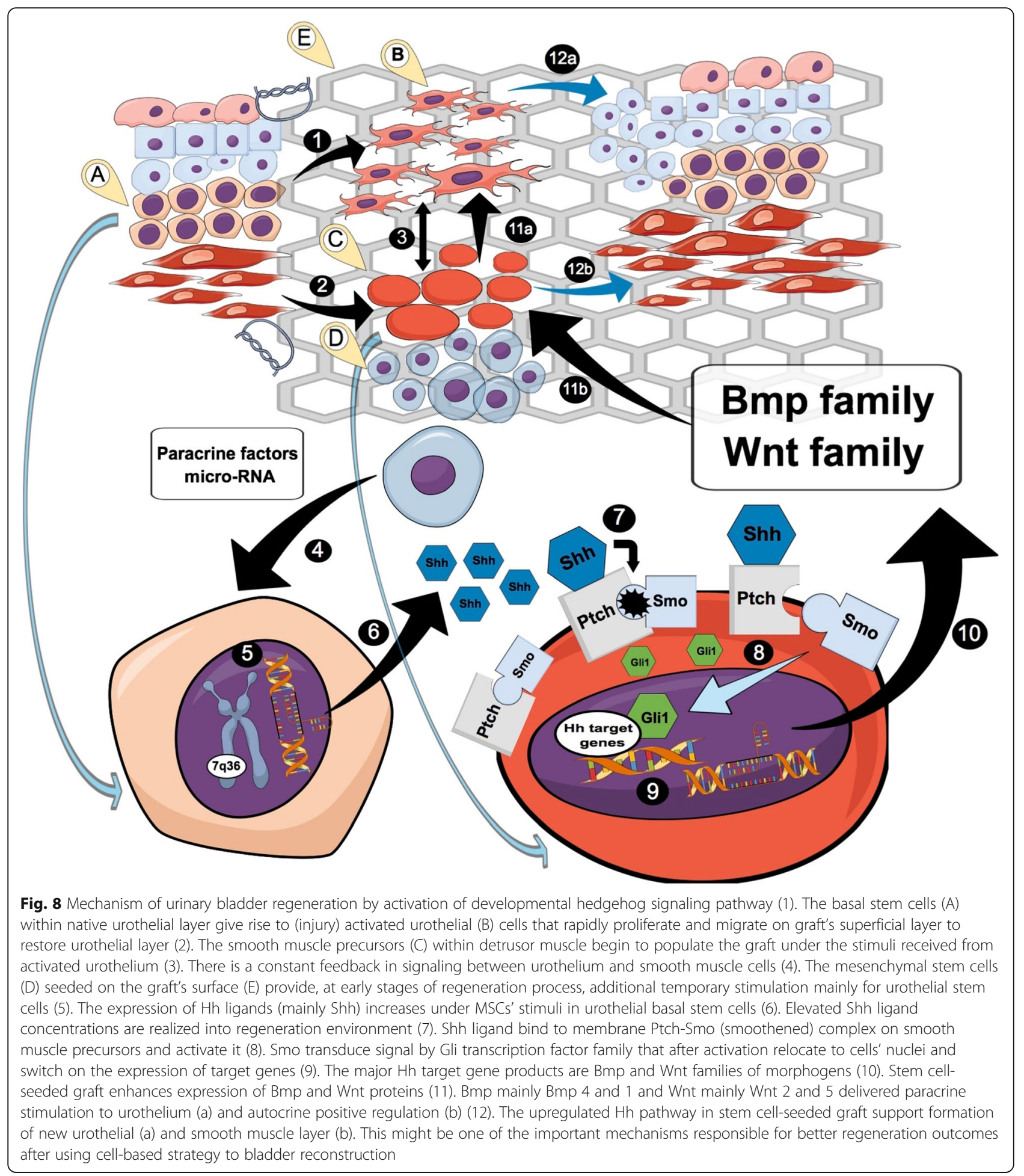

new signaling architecture results in the activation of regenerative mechanisms that were previously switched off at early ontogenesis stages. The signaling pathways modulated by ASCs were continuously changing after the reconstruction procedure, revealing an immense complicated nature of the healing process. This could explain why we have not to date succeeded in inducing regeneration by applying scaffolds with an incorporated artificial selection of growth factors [18].

It is important to determine how long implanted ASCs stay active within the graft site [5]. Evaluated ASCs delivered a long lasting stimulation that guided new 
bladder wall formation. Although changes in the profile of upregulated pathways were present even at 180 days after transplantation, the number of pathways modulated by ASCs gradually decreased. The explanation for this phenomenon is time limited ASCs survival, which differ from 1 week to 6 months. The hierarchical cluster analysis for long 180 days follow-up shows that the length of implantation had a greater influence on the gene expression in the tissue and not whether the BAM was seeded with ASC or not. In the shorter 7, 30, and 90 days follow-up, implanted ASCs have great impact on gene expression in tissue-engineered bladders. The hierarchical cluster analysis clearly shows that ASCs accelerate the healing of tissue-engineered bladder. The gene expression profile in bladders reconstructed using BAM seeded with ASCs at day 7 was comparable to the profile observed in the bladders reconstructed using BAM only at day 30, while the profile of gene expression observed in the bladders reconstructed using BAM seeded with ASCs at day 30 to the that observed in the bladders reconstructed using BAM only at day 90 .

Histological analysis of reconstructed bladders revealed well-regenerated urothelial and muscle layers after final follow up. Urothelium as an epithelial compartment displayed better self-restoration ability than muscle layers. Proper smooth muscle layers were only found in the bladders reconstructed with cellular graft. Local signaling mainly derived from local resident smooth muscle progenitor cells is believed to drive MSCs towards a smooth muscle fate [19]. Activation of the hypertrophic cardiomyopathy pathway might indicate locally increased smooth cell migration mediated by the RAS-MAPK pathway. Autocrine signaling through RAS suppressed apoptosis and stimulated many types of smooth muscle cells [20].

Bladder regeneration requires adequate distribution of key morphogenetic signals transmitted by a number of paracrine pathways activated in a very coordinated manner [4]. The chemokine signaling pathway involved in cell communication was activated at the early stages of bladder wall regeneration. This result suggested an intensified intercellular communication in tissue-engineered urinary bladder, especially during the initial phase of bladder healing. Complementary downregulation of cytokine-cytokine receptor interaction pathways indicated an unspecified boost of elementary signaling transmission triggered by ASCs. By contrast, pathways regulating compartmentalization of destined cellular phenotypes were upregulated at distant regeneration time points that corresponded to a late remodeling period.

Interesting results from microarray analyses were significant changes in olfactory transduction pathways between bladders reconstructed with BAM seeded with or without ASCs during early regenerative stages. This might be an indicator of intensified communication between the nervous and endocrine systems mediated by interstitial cells. Kang et al. announced expression of olfactory receptor-mediated chemoreception in nonolfactory systems like interstitial cells of Cajal in the bladder [21]. The role of this cell population during bladder regeneration is however poorly understood. Nevertheless, as our study showed, their activity should be further explored. Determining novel research directions is an essential advantage of microarray analysis, which allows us to receive an overview of the signaling network guiding bladder regeneration. As induced bladder regeneration is a dynamic scenario with timedepended regulatory switches, summarizing microarray analysis helped to identify overriding pathways and specify their activity period. This data should mitigate gaining control over this process and in turn make its outcomes predictable.

Before translation of tissue-engineered based technologies into reconstructive urology, there is a need to better understand the host's immune response towards implanted cell-seeded grafts. Changes in the allograft rejection pathway, which was pivotal for long-term graft acceptance, were registered 30 days after bladder augmentation. At this time, ASCs simultaneously modified several major immunomodulatory pathways. This finding suggests that the breaking point for immune events are by end of the first month for tissue-engineered bladder reconstruction and this might even serve as a benchmark for further research. MSCs are characterized by their immunosuppressive properties, being one of the mechanisms by which MSCs exert their reparative benefits. MSCs act as immunomodulatory agents that silence immune responses locally, creating areas of attenuated inflammatory reactions and in turn hamper fibrosis [4]. TGF-beta is a major coupling factor between fibrotic and inflammatory processes [22]. Applied ASCs decreased the expression of TGF-beta pathway genes already at the initial healing stage and thus determined a favorable fate of reprogramming bladder wall tissue towards regeneration.

Significant differences in the activation of Hippo signaling pathway, known as the Salvador/Warts/Hippo pathway, that controls organ size through the regulation of cell proliferation and apoptosis were observed in bladders reconstructed with and without ASCs. There is an increasing amount of evidence to suggest that the Hippo signaling pathway plays a critical role in regulating organ regeneration across different species [23]. Similarly, JakSTAT and PI3K-Akt signaling pathways were differentially expressed in bladders reconstructed with and without ASCs. The PI3K-Akt signaling pathways play central regulatory roles in MSCs survival, proliferation, migration, angiogenesis, cytokine production, and 
differentiation [24]. JAK-STAT pathway regulates myogenic differentiation [25]. Our study confirmed that they are also directly related to urinary bladder regeneration.

What particularly concerned us was the registered activity of different oncogenic pathways during bladder wall regeneration. In context of an unresolved discussion about carcinogenesis risk increased by chronic or recurrent bladder infections, this finding is of importance [26]. Each urinary bladder infection ended with spontaneous bladder healing. Therefore, repeating sequences of bladder lining disruption and regeneration might lead to uncontrolled oncogenic pathway activation.

The current study provides also a very important demonstration of Hh (Hedgehog) signaling role during ASCinduced bladder regeneration and contribution made by Hh signaling to early stages of bladder healing. Landmark study of Baskin et al. outlined the framework of signaling hierarchy during urinary bladder organogenesis [27]. They made the remarkable observation that smooth muscle differentiation from bladder mesenchyme depended on signals that originated in the urothelium. As we believed that bladder wall regeneration is similar process to bladder organogenesis, comparable set of evolutionary conserved signaling pathways should be engaged to both processes. Analogs to urinary bladder development, key morphogenetic signals mediated by Bmp and Wnt proteins are required to obtain proper differentiation pattern and subsequent compartmentalization of distinct cell types [28]. Postnatal quiescence of urothelium is likely to be a default state in the absence of a pro-proliferative signaling rapidly generated within injury site [29]. The disruption of the urothelial and smooth muscle layer after urinary bladder augmentation with in vitro constructed graft initiate acquisition of a highly proliferative and migratory phenotype by urothelial cells [30]. Shin et al. identified stem cells with basal layer of urothelium that shifted from near-quiescence to a highly proliferative state in response to epithelial injury [31]. Described cell population was marked by extensive Hh expression. This report is consistent with our findings revealing activation of $\mathrm{Hh}$ pathway during the first week after bladder reconstruction. Peyton et al. also demonstrated that bladder response to subtotal cystectomy resulted in urothelial cell activation and rapid proliferation of this cell population within the first 3 days [32]. In that case, immunofluorescence-based measurement showed elevated expression of SHH, GLi-1, and BMP-4 by the end of the first week after injury. Identical expression pattern was confirmed in our study by microarray and real-time PCR analysis. We hypothesize that the gradient of $\mathrm{Hh} \mathrm{li-}$ gands, such as Shh (Sonic hedgehog), Ihh (Indian hedgehog), and Dhh (Desert hedgehog), might be formed at the border between native bladder wall and sutured in vitro constructed graft within the first week after augmentation. Constituted Hh ligands' gradient might in turn attract urothelial cell to migrate into the external graft's margin and populate the BAM scaffold. Convergence of research results focused on the role of Hh during bladder regeneration gathered using different models indicates that this cascade is early activated, drives initial cellular events, and thus might determine the fate of bladder regeneration [31, 33-35]. Therefore, in our opinion, it is currently the best known target for future therapies oriented to support the bladder regeneration. We previously demonstrated that bladder wall regeneration reached better quality within grafts seeded with MSCs isolated from adipose tissue as well as bone marrow compared to unseeded grafts $[4,5,12]$. Both urothelial and smooth muscle cells were better developed under stimulation derived from MSCs seeded on scaffolds applied for experimental bladder reconstruction. In group with transplanted ASCs, the activation Hh pathway was significantly increased at 7 and 30 day. Transplanted stem cells modulated regeneration environment by their wide paracrine activity. MSCs apparently activated intrinsic regeneration mechanisms that were silenced during ontogenesis. Despite years of research, the discussion about the exact mechanisms that are involved in pronounced regeneration after MSC transplantation continues. Identification of these signaling routes would be helpful during designing bioactive biomaterials with incorporated growth factors. Recruiting of Hh pathway, triggered by ASCs, might partially explain proregenerative properties of these cells and open new perspective for the therapeutic solutions for bladder wall regeneration. Wang et al. recently reported that artificial supplementation of injured site of the ventricular epicardium with Shh stimulated both epicardium and neighbor cardiomocyte regeneration [36]. As major cellular regeneration mechanisms are universal in different tissue types upregulated Hh pathway might analogously promote detrusor regrowth. Observed better development of smooth muscle layer within cell seeded grafts might be linked to enhanced smooth muscle proliferation mediated by Hh cascade. Hh signaling was documented to be involved in controlling of smooth muscle proliferation in many organs [37-39]. Following research of Caubit et al., transcription factor teashirt 3 (Tshz3) is an important regulator of smooth muscle differentiation in urinary tracts mediated by Shh and Bmp4 [40]. If Hh pathway plays essential role during bladder wall regeneration induced by ASCs, the further matter of discussion should be signaling hierarchy responsible for this effect. ASCs may either directly or indirectly elevate $\mathrm{Hh}$ expression within regenerating bladder wall. Therefore, ASCs might selectively upregulate expression of $\mathrm{Hh}$ ligands in urothelial cells or mobilize and increase 
survival of injury-activated urothelial cells that naturally express Hh ligands. We opt for dominant importance of unselective mechanism as ASCs exhibit broad paracrine activity influencing simultaneously multiple signaling cascades. Hh pathway establishes three-level signal transmission between ASCs, urothelial cells, and smooth muscle precursors. Molecular mechanism governing upregulation of Hh by ASCs is still poorly understood. The abundant growth factors are believed to be involved in this signaling but as Hyun et al. recently reported microRNA-mediated regulation might be important player in this field [41]. ASC-derived stimulation was temporary and maintained over the first month. After the 30th day, the "booster effect" exerted by MSC was depleted, and hence, there was no difference in Hh expression in groups with or without cells. The lack of long-lasting effect after cell-based bladder regeneration hampers translation of this approach into clinics. The harsh graft's microenvironment with inflammation, ischemia, oxidative stress, and mechanical stress contributes to poor stem cell survival [5]. The confirmed upregulation of Hh pathway by transplanted ASCs led to increased synthesis of Bmp and Wnt family proteins. Bmp function in concert with Wnt and their involvement in reconstitution of pivotal bladder component including urothelial and smooth muscle layers were previously demonstrated $[34,35]$. Shh seems to be a major Hh ligand positively impacting Bmp and Wnt expression in smooth muscle precursor or mesenchymal stromal cells during bladder organogenesis and tissue injury. The Bmp4 is a product of one of the major target gene regulated by $\mathrm{Hh}$ and functions in organ of endodermal origin such as the urinary bladder as a potent morphogen involved in spontaneous regeneration process [42]. Mysorekar et al. described high expression of Bmp4 receptor in progenitor urothelial cells, identified after urothelium response to uropathogenic infection [34]. They concluded that the Bmp4 was a major protein regulating urothelium regeneration. Considering studies' outcomes discussing Bmp involvement in smooth muscle regeneration, its function seems to be more complex as Bmp might be crucial factor organizing signaling within urothelium-smooth muscle axis. Bmp2 and Bmp4 expression was increased only at day 7 that corresponds to the period of rapid urothelial proliferation and final stages of graft's reepithelialization. The stimulation derived from ASCs was most likely missing at further regeneration stages, and in this situation, any changes in expression pattern between cellular and acellular scaffold were not detected.

The existing model of bladder wall regeneration assumes profound signaling feedback between urothelial and smooth muscle precursors. Interestingly, Shin et al. postulated the existence of profound signaling feedback involving Hh pathway, between urothelial and bladder stromal cells, that was responsible for bladder cancer progression [43]. Therefore, elucidation of urinary bladder regeneration will shed in turn also new light on carcinogenesis within bladder wall. This knowledge will help to identify ambiguous associations between signaling cascades driving bladder regeneration but, in different circumstances, promoting cancer growth.

\section{Conclusions}

In conclusion, the study provided the unequivocal evidence that stem cells changed the healing milieu in tissue-engineered urinary bladder and indicated pathways that can be associated with the regeneration process triggered by stem cells. Of particular interest are pathways being a key regulator of embryonic development, involved in multiple processes including cell fate determination, tissue patterning, and morphogenesis as well as regulation of adult stem cell renewal, maintenance of homeostasis, and regeneration of adult tissues, such us Hedgehog, TGF- $\beta$, Jak-STAT, PI3-Akt, and Hippo signaling pathways. Within our study, we have demonstrated that transplanted into reconstructed bladder ASCs exert their regenerative effect by the upregulation of Hh pathway. Nevertheless, this upregulation took place shortly after the reconstruction and was gradually silenced afterwards. This might be an explanation of poor distant results of tissue engineering approach to bladder reconstruction even though we tried to use different stem cells to induce regeneration mechanisms. This results show that Hh pathway can serve as a potential target for induced urinary bladder regeneration.

\section{Additional files}

Additional file 1: Table S1. Primer and probe sequences. (DOC 89 kb) Additional file 2: Table S2. Involvement of Differentially Expressed Genes (DEGs) between bladders reconstructed using BAM seeded with or without ASCs in KEGG pathways at day 7 (A), 30 (B), 90 (C), and 180 (D) follow up. (DOC $127 \mathrm{~kb}$ )

Additional file 3: Table S3. Involvement of Differentially Expressed Genes (DEGs) between bladders reconstructed using BAM seeded with or without ASCs in WikiPathways. (DOC $183 \mathrm{~kb}$ )

Additional file 4: Table S4. Gene Ontologies (GO) enrichment analysis on DEGs between bladders reconstructed using BAM seeded with or without ASCs at 7, 30, 90, and 180 days postoperatively. Selected GO critical for urinary bladder healing with $p<0.05$ are presented. (DOC $351 \mathrm{~kb}$ )

Additional file 5: Figure S1. Flow cytometry analysis for the expression of cell surface antigens: CD11b, CD29, CD31, CD44, CD45, and CD90. The red histograms show staining with isotype controls, and the gray histograms represent staining with the specified surface marker antibody. The experiment was performed in three replicates. The results from one representative ASCs immunophenotype analysis are shown (A).

Differentiation potential of ASCs: a positive Oil Red O staining of lipid vacuoles after 21 days of adipogenic induction, bar $200 \mu \mathrm{m}$ (B); Alcian blue staining of proteoglycans after 14 days of chondrogenic induction, bar $100 \mu \mathrm{m}(\mathrm{C})$; Alizarin red staining of mineral deposits after 21 days of osteogenic induction, bar $100 \mu \mathrm{m}$ (D); ASCs cultured in standard medium 
remained undifferentiated (E-G), bar $200 \mu \mathrm{m}, 100 \mu \mathrm{m}$ and $100 \mu \mathrm{m}$, respectively. Bladder Acellular Matrix (BAM) $(\mathrm{H})$ and BAM seeded with Adipose Derived Stem Cells (ASCs) (I,J). ASCs cultivated on BAM for 7 days form a homogenous layer $(1, J)$. Scanning electron microscope, bar 2 and 10 um. (TIF $2826 \mathrm{~kb}$ )

Additional file 6: Figure S2. Representative histological images of urothelium (A-C) and smooth muscle regeneration (D-F) and inflammatory response (G-I) in tissue-engineered urinary bladders. Normal urothelium with 5 cell layers (A), normal urothelium with $\leq 4$ cell layers (B), lack of urothelium (C), smooth muscle layer with regular (D) and irregular (E) fiber arrangement, incomplete smooth muscle layer (F), lack of inflammatory response $(G)$, moderate $(H)$, and intense (I) inflammatory response are presented. Light microscope, bar 100um, 400 um. Histological analysis of urothelium $(\mathrm{J})$ and smooth muscle regeneration $(\mathrm{K})$ and inflammatory response $(L)$ in tissue-engineered urinary bladders at 7, 30, 90 , and 180 days postoperatively. Urothelium was assessed as 3 normal $\geq 5$ layers, 2 normal $\leq 4$ layers, 1 changed by inflammatory reaction, 0 lack. Smooth muscle was assessed as 3 normal, 2 irregular arrangement, 1 incomplete, 0 lack. Inflammatory reaction was assessed as 3 very intense, 2 intense, 1 moderate, and 0 lack. The histological analyses were performed in three replicates for each group $(n=24)$. (TIF $18353 \mathrm{~kb})$

\section{Abbreviations}

ASCs: Adipose tissue-derived mesenchymal stromal cells; BAM: Bladder acellular matrix; Bmp: Bone morphogenetic proteins; DEG: Differentially expressed genes; Dhh: Desert Hedgehog; Gli: Glioma-associated oncogene homolog 1; Ihh: Indian Hedgehog; Jak-STAT: Janus kinase/signal transducers and activators of transcription; MSCs: Mesenchymal stem cells; PI3Akt: Phosphatidylinositol 3-kinase/Akt; Ptch1: Patched 1; Rab23: Ras-related protein Rab-23; Shh: Sonic Hedgehog; Smo: Smoothened; TGF$\beta$ : Transforming growth factor $\beta$

\section{Acknowledgements}

Not applicable.

\section{Funding}

This research was supported by a grant from the Polish National Science Center, decision no. DEC-2012/07/D/NZ1/00854.

\section{Availability of data and materials}

The microarray gene expression data have been deposited at the NCBI Gene Expression Omnibus. The data are under accession number GEO: GSE103572.

\section{Authors' contributions}

MP contributed in the conception and design, collection and/or assembly of data, analysis and interpretation of data, manuscript writing, and the final approval of the manuscript. MR took part in the collection and/or assembly of data as well as in the analysis and interpretation of data. AJ, DB, KW, and LB have a hand in the collection and/or assembly of data. JA helped in the analysis and interpretation of data and manuscript writing. TD did the critical revision. All authors read and approved the final manuscript.

\section{Ethics approval and consent to participate}

The study was carried out in strict accordance with recommendations from the Guide for the Care and Use of Laboratory Animals of the National Institutes of Health. The protocol was approved by the Nicolaus Copernicus University Ethics Committee (no. 46/2012).

\section{Consent for publication}

Not applicable.

\section{Competing interests}

The authors decelerate that they have no competing interest.

\section{Publisher's Note}

Springer Nature remains neutral with regard to jurisdictional claims in published maps and institutional affiliations.
Received: 22 March 2019 Revised: 13 May 2019

Accepted: 14 May 2019 Published online: 13 June 2019

\section{References}

1. Adamowicz J, Pokrywczynska M, Van Breda SV, Kloskowski T, Drewa T. Concise review: tissue engineering of urinary bladder; we still have a long way to go? Stem Cells Transl Med. 2017;6:2033-43.

2. Drewa T, Adamowicz J, Sharma A. Tissue engineering for the oncologic urinary bladder. Nat Rev Urol. 2012;9:561-72.

3. Atala A, Bauer SB, Soker S, Yoo JJ, Retik AB. Tissue-engineered autologous bladders for patients needing cystoplasty. Lancet. 2006;15:1241-6.

4. Pokrywczynska M, Jundzill A, Bodnar M, Adamowicz J, Tworkiewicz J, Szylberg $L$, et al. Do mesenchymal stem cells modulate the milieu of reconstructed bladder wall? Arch Immunol Ther Exp. 2013;61:483-93.

5. Pokrywczynska M, Jundzill A, Rasmus M, Adamowicz J, Balcerczyk D, Buhl M, et al. Understanding the role of mesenchymal stem cells in urinary bladder regeneration-a preclinical study on a porcine model. Stem Cell Res Ther. 2018;9:328.

6. Coutu DL, Mahfouz W, Loutochin O, Galipeau J, Corcos J. Tissue engineering of rat bladder using marrow-derived mesenchymal stem cells and bladder acellular matrix. PLoS One. 2014;9:e111966-21.

7. Jack GS, Zhang R, Lee M, Xu Y, Wu BM, Rodriguez LV. Urinary bladder smooth muscle engineered from adipose stem cells and a three dimensional synthetic composite. Biomaterials. 2009;30:3259-70.

8. Kajbafzadeh AM, Tourchi A, Mousavian AA, Rouhi L, Tavangar SM, Sabetkish N. Bladder muscular wall regeneration with autologous adipose mesenchymal stem cells on three- dimensional collagen-based tissueengineered prepuce and biocompatible nanofibrillar scaffold. J Pediatr Urol. 2014;10:1051-8.

9. Leite MT, Freitas-Filho LG, Oliveira AS, Semedo-Kuriki P, Laks M, Arias VE, et al. The use of mesenchymal stem cells in bladder augmentation. Pediatr Surg Int. 2014;30:361-70

10. Pokrywczynska M, Jundzill A, Adamowicz J, Kowalczyk T, Warda K, Rasmus $\mathrm{M}$, et al. Is the poly (L-lactide-co-caprolactone) nanofibrous membrane suitable for urinary bladder regeneration? PLoS One. 2014;9:e105295.

11. Sharma AK, Bury MI, Marks AJ, Fuller NJ, Meisner JW, Tapaskar N, et al. A nonhuman primate model for urinary bladder regeneration using autologous sources of bone marrow-derived mesenchymal stem cells. Stem Cells. 2011:29:241-50.

12. Pokrywczynska M, Jundzill A, Warda K, Ramus M, Adamowicz J, Bodnar M, et al. Does mesenchymal stem cell source influence smooth muscle regeneration in tissue engineered urinary bladder? Cell Transplant. 2017;26: 1780-91.

13. National Research Council. Guide for the care and use of laboratory animals. 8th ed. Washington, DC: the National Academies Press; 2011.

14. Huang DW, Sherman BT, Lempicki RA. Systematic and integrative analysis of large gene lists using DAVID Bioinformatics Resources. Nature Protoc. 2009a; 4:44-57.

15. Huang DW, Sherman BT, Lempicki RA. Bioinformatics enrichment tools: paths toward the comprehensive functional analysis of large gene lists. Nucleic Acids Res. 2009b;37:1-13.

16. Pokrywczynska M, Czapiewska M, Jundzill A, Bodnar M, Balcerczyk D, Kloskowski T, et al. Optimization of porcine urothelial cell cultures: best practices, recommendations, and threats. Cell Biol Int. 2016a;40:812-20.

17. Pokrywczynska M, Balcerczyk D, Jundzill A, Gagat M, Czapiewska M, Kloskowski T, et al. Isolation, expansion and characterization of porcine urinary bladder smooth muscle cells for tissue engineering. Biol Proced Online. 2016b;18:17.

18. Nuininga JE, Koens MJ, Tiemessen DM, Oosterwijk E, Daamen WF, Geutjes PJ, et al. Urethral reconstruction of critical defects in rabbits using molecularly defined tubular type I collagen biomatrices: key issues in growth factor addition. Tissue Eng Part A. 2010;16:3319-28.

19. Yang $B$, Zheng $J H$, Zhang $Y Y$. Myogenic differentiation of mesenchymal stem cells for muscle regeneration in urinary tract. Chin Med J. 2013;126: 2952-9.

20. Muto A, Fitzgerald TN, Pimiento JM, Maloney SP, Teso D, Paszkowiak JJ, et al. Smooth muscle cell signal transduction: implications of vascular biology for vascular surgeons. J Vasc Surg. 2007:45:A15-24.

21. Kang $N$, Kim H, Jae $Y$, Lee $N$, Ku CR, Margolis F, et al. Olfactory marker protein expression is an indicator of olfactory receptor-associated events in non-olfactory tissues. PLoS One. 2015;10:e0116097. 
22. Meng XM, Nikolic-Paterson DJ, Lan HY. TGF- $\beta$ : the master regulator of fibrosis. Rev Nephrol. 2016;12:325-38.

23. Juan WC, Hong W. Targeting the hippo signaling pathway for tissue regeneration and cancer therapy. Genes (Basel). 2016;7:55.

24. Chen J, Crawford R, Chen C, Xiao Y. The key regulatory roles of the PI3K/Akt signaling pathway in the functionalities of mesenchymal stem cells and applications in tissue regeneration. Tissue Eng Part B Rev. 2013;19(6):516-28.

25. Jang YN, Baik EJ. JAK-STAT pathway and myogenic differentiation. JAKSTAT. 2013;2:e23282.

26. Michaud DS. Chronic inflammation and bladder cancer. Urol Oncol. 2007;25: 260-8.

27. Baskin LS, Hayward SW, Young P, Cunha GR. Role of mesenchymal-epithelial interactions in normal bladder development. J Urol. 1996;156:1820-7.

28. Cao M, Tasian G, Wang MH, Liu B, Cunha G, Baskin L. Urothelium-derived sonic hedgehog promotes mesenchymal proliferation and induces bladder smooth muscledifferentiation. Differentiation. 2010;79:244-50.

29. Balsara ZR, Li X. Sleeping beauty: awakening urothelium from its slumber. Am J Physiol Renal Physiol. 2017;312:F732-43.

30. Birder LA, de Groat WC. Mechanisms of disease: involvement of the urotheliumin bladder dysfunction. Nat ClinPract Urol. 2007;4:46-54.

31. Shin K, Lee J, Guo N, Kim J, Lim A, Qu L, et al. Hedgehog/Wnt feedback supports regenerative proliferation of epithelial stem cells in bladder. Nature. 2011;472:110-4.

32. Peyton CC, Burmeister D, Petersen B, Andersson KE, Christ G. Characterization of the early proliferative response of the rodent bladder to subtotal cystectomy: a unique model of mammalian organ regeneration. PLoS One. 2012;7:e47414.

33. DeSouza KR, Saha M, Carpenter AR, Scott M, McHugh KM. Analysis of the Sonic Hedgehog signaling pathway in normal and abnormal bladder development. PLoS One. 2013;8:e53675.

34. Mysorekar IU, Isaacson-Schmid M, Walker JN, Mills JC, Hultgren SJ. Bonemorphogenetic protein 4 signaling regulates epithelial renewal in the urinary tract in response to uropathogenic infection. Cell Host Microbe. 2009;5:463-75

35. Shin K, Lim A, Zhao C, Sahoo D, Pan Y, Spiekerkoetter E, et al. Hedgehog signaling restrains bladder cancer progression by eliciting stromal production of urothelial differentiation factors. Cancer Cell. 2014a;26:521-33.

36. Wang J, Cao J, Dickson AL, Poss KD. Epicardial regeneration is guided bycardiac outflow tract and Hedgehog signalling. Nature. 2015;522:226-30.

37. Langiewicz M, Schlegel A, Saponara E, Linecker M, Borger P, Graf R, et al. Hedgehog pathway mediates early acceleration of liver regeneration induced by a novel two-staged hepatectomy in mice. J Hepatol. 2017;66: 560-70.

38. Sriperumbudur A, Breitzig M, Lockey R, Kolliputi N. Hedgehog: the key to maintaining adult lung repair and regeneration. J Cell Commun Signal. 2017;11:95-6.

39. Dunaeva $\mathrm{M}$, Waltenberger J. Hh signaling in regeneration of the ischemic heart. Cell Mol. 2017;74:3481-90.

40. Caubit X, Lye CM, Martin E, Coré N, Long DA, Vola C, et al. Teashirt 3 is necessary for ureteral smooth muscledifferentiation downstream of $\mathrm{SHH}$ and BMP4. Development. 2008;135:3301-10.

41. Hyun J, Choi SS, Diehl AM, Jung Y. Potential role of Hedgehog signaling and microRNA-29 in liver fibrosis of IKKß-deficient mouse. J MolHistol. 2014; 45:103-12.

42. Liu B, Feng D, Lin G, Cao M, Kan YW, Cunha GR, et al. Signalling molecules involved in mouse bladder smooth muscle cellular differentiation. Int J Dev Biol. 2010;54:175-80.

43. Shin K, Lim A, Odegaard Jl, Honeycutt JD, Kawano S, Hsieh MH, et al. Cellular origin of bladder neoplasia and tissue dynamics of its progression toinvasive carcinoma. Nat Cell Biol. 2014b;16:469-78.

Ready to submit your research? Choose BMC and benefit from:

- fast, convenient online submission

- thorough peer review by experienced researchers in your field

- rapid publication on acceptance

- support for research data, including large and complex data types

- gold Open Access which fosters wider collaboration and increased citations

- maximum visibility for your research: over $100 \mathrm{M}$ website views per year

At BMC, research is always in progress.

Learn more biomedcentral.com/submissions 